\title{
Research Progress of HSP70 and Chronic Atrophic Gastritis
}

\author{
Chen-Chen Zhou, Rong Ji and Li-Qiang Huang* \\ Zhangjiagang TCM Hospital, Affiliated to Nanjing University of Chinese Medicine, China
}

*Corresponding author: Li-Qiang Huang, Zhangjiagang TCM Hospital Affiliated to Nanjing University of Chinese Medicine, Jiangsu 215600, China

To Cite This Article: Chen-Chen Zhou, Rong Ji, Li-Qiang Huang, Research Progress of HSP7O and Chronic Atrophic Gastritis. Am J Biomed Sci \& Res. 2021 - 12(3). AJBSR.MS.ID.001753. DOI: 10.34297/AJBSR.2021.12.001753.

Received: 眥 March 10, 2021; Published: 阱 March 31, 2021

\begin{abstract}
Chronic atrophic gastritis is still one of the main diseases threatening human health, and CAG patients have an increased risk of gastric cancer. Heat shock protein 70 protects the gastric mucosa by inhibiting the activation of protein kinase JNK and NF-kB inflammatory pathways in chronic atrophic gastritis. At present, HSP70 has been clinically used in the treatment and prevention of chronic atrophic gastritis. This article reviews the research on the relationship between HSP70 and chronic atrophic gastritis in recent years.
\end{abstract}

Key words: Heat Shock Protein 70; Chronic Atrophic Gastritis

\section{Introduction}

Chronic atrophic gastritis (CAG) means that the epithelial cells of the gastric mucosa are repeatedly and long-term invaded, which firstly causes local inflammation of the gastric mucosa, which in turn affects the blood circulation of the gastric mucosa and destroys the gastric mucosal barrier. The proper glands began to shrink, the mucosa became thinner and even accompanied by metaplasia and dysplasia of the intestinal epithelium. CAG is the result of multiple factors, and helicobacter pylori infection is the main pathogenic factor. Studies have shown that the gastric mucosa activates a variety of defense systems when stimulated by food, smoking, alcohol, helicobacter pylori and other factors. The heat shock response system is one of the defense functions in the acute phase, and the most important type of heat shock protein Heat shock proteins (HSP) is a family of HSP70 with a molecular weight of about 70kD. It reduces stress by assisting in protein repair, inhibiting oxidative damage, and regulating gastric mucosal blood flow.

\section{HSP70 Classification and Molecular Structure}

The HSP70 family is the most conserved, most important and abundant class of heat shock proteins, which consists of 21 proteins. HSP70 is mainly expressed in the cytoplasm of lamina propria glandular epithelial cells and mucosal epithelial cells, which can be subdivided into 4 types: a. HSP72, namely inducible HSP70, is expressed in large quantities in stress response and distributed in the cytoplasm and nucleus

b. HSP73, structural HSP70, can be expressed in all cells and distributed in the cytoplasm and nucleus under heat guidance

c. GRP78, distributed in the endoplasmic reticulum

d. GRP75, distributed in the mitochondria. among them. HSP73, GRP75, and GRP78 are present in non-stressed normal cells and can be expressed in normal cells, while HSP72 is only present in stressed cells [1].

HSP70 protein is divided into three domains: the aminoterminal (1-383) ATP binding domain (ATP-BD), which has ATPase activity and can hydrolyze ATP, which is more conservative than the C-terminal; The peptide binding domain (PBD) in the central region (384-542) can bind to new peptides and damaged proteins; the highly conserved terminal sequence of the carboxyl terminal (543-646) can affect the amount of HSP70 mRNA translation [2].

\section{Expression and regulation of HSP70}

Theregulation of geneexpressionin theHSP70 family is a complex process, and the specific mechanism is not yet fully understood. 
Munro et al. believe that the expression of HSP70 is closely related to the Ub-independent lysosomal protein degradation system. They believe that under normal circumstances, HSF1 is ubiquitinated as an inactive monomer. Under stress, the production of a large number of denatured proteins in the cell activates the Ub-mediated protein degradation system, the level of free Ub decreases, the binding to HSF1 decreases, and the free monomer HSF1 increases. Somatic HSF1 forms an active trimeric form, is phosphorylated and enters the nucleus, binds to HSE, and induces the transcription of HSP70 gene under the action of RNA polymerase. As the denatured protein is largely degraded by hydrolase, and the cells continue to synthesize new Ub, HSF recombines with Ub and loses its activity. Terminate the transcription of HSP70 gene [3].

Under normal circumstances, the half-life of HSP70mRNA is $15-30 \mathrm{~min}$, and its half-life is as long as $4 \mathrm{~h}$ under heat stress. At the same time, HSP70mRNA is translated in large amounts, and other mRNAs in the cell are not degraded but translation stops.

\section{The biological function of HSP70}

\section{Molecular chaperone function}

Molecular chaperones are a large class of proteins in cells, a family composed of unrelated proteins, which mediate the correct distribution of other proteins, but do not themselves become a component of the final functional structure. HSP is such a class that assists in the assembly and assembly of intracellular molecules. A protein that assists in protein folding. The molecular chaperone functions of HSP mainly include:

a. participate in protein folding

b. promote the degradation of misfolded proteins

c. help proteins complete intracellular transmembrane transfer

This speeds up the synthesis of denatured proteins from normal proteins, maintains the stability of cellular proteins, and improves the tolerance of cells to stressors.

\section{Anti-Apoptosis}

Apoptosis means that when cells are subjected to certain physiological and pathological stimuli, in order to maintain the stability of their internal environment, the cells activate the expression of related genes to cause the cells to self-destruct and die. Studies have shown that HSP70 can regulate and inhibit cell apoptosis at multiple levels. For example, HSP70 can inhibit the release of cytochrome $\mathrm{c}$ from mitochondria, thereby inhibiting the activation of the caspase pathway; inhibit stress-activated protein kinase (c-Jun N-terminal kinase, JNK) and p38 mitogen-activated protein kinase (p38 MAPK); inhibit the expression of apoptotic genes p53 and Bax; inhibit the generation of oxygen free radicals; inhibit apoptotic protease activity factors and apoptosis-inducing factors.

\section{Improve Cell Tolerance}

HSP70 inhibits the activity of nuclear factor kappa B (NF$\mathrm{kB}$ ) promoters, so that the inflammatory factors tumor necrosis factor- $\alpha$, interleukin (IL)- 6 and IL- $1 \beta$ downstream of the signaling pathway the product is reduced and the cell inflammatory reaction process is reduced.

\section{Anti-Oxidant Stress}

Under stress conditions, the body produces a large number of oxygen free radicals, which act on cells, mitochondria, lysosomes and other biofilms, and reduce the ability to regulate $\mathrm{Ca} 2+$ in and out of cells, causing cytotoxicity. HSP70 inhibits the key enzyme that produces oxygen free radicals, namely nicotinamide adenine dinucleotide phosphate oxidase, inhibits the production of oxygen free radicals, and can also increase the level of endogenous superoxide dismutase- 2 and speed up the removal of oxygen free radicals. The lipid peroxidation reaction induced by free radicals participates in the process of gastric mucosal damage caused by various reasons. It enhances the scavenging of free radicals and reduces the lipid peroxidation reaction, which is one of the roles of HSP70 in protecting the gastric mucosa.

The function of HSP70 has the following characteristics:

a. The level of HSP70 is low under normal conditions, but its expression is significantly increased under stress;

b. HSP70 mainly exists in the cytoplasm at ordinary times, and HSP70 quickly enters the nucleus under stress;

c. A variety of factors can induce the mass production of HSP70, which has universal stress response, and its induced synthesis is regulated at the two levels of transcription and translation;

d. HSP70 can improve the anti-stress ability of cells. For example, the amount of HSP70 produced is positively correlated with the heat tolerance of cells.

\section{The Effect of HSP70 on Chronic Atrophic Gastritis}

HSP70 exists in the gastric mucosa. The increased expression of HSP can cause the up-regulation of the expression of reduction oxidase 1 and oxidative synthetase, and reduction oxidase 1 increases the production of prostaglandin E2, which has a protective effect on the mucosa, while the catalytic product of oxidative synthetase is oxidized. Nitrogen can increase the blood flow of the gastric mucosa and is also a protective effect on the gastric mucosa. Studies have shown that HSP70 can inhibit mucosal inflammation and promote the healing of ulcers. At the same time, HSP70 can resist the effects of Helicobacter pylori-induced gastric mucosal cell apoptosis, weakened epithelial cell migration, reduced local blood flow, and inhibition of neovascularization. Wu Yong Mei et al. [4] showed that HSP70 is expressed in superficial gastritis, CAG, and gastric cancer tissues. HSP70 functions as a molecular chaperone, 
stabilizes protein conformation, accelerates the degradation of abnormal proteins, can maintain cell stability, improve cell survival, and accelerate the conversion of denatured proteins, thereby accelerating the expression of HSP70. Other studies have shown that HSP70 can inhibit cell apoptosis, combat the damage of non-steroidal anti-inflammatory drugs to gastric mucosa, and reduce the cytotoxicity of Helicobacter pylori. HSP70 mediates cell apoptosis at multiple levels. By inhibiting the activation of protein kinase JNK (a regulator of cell apoptosis), it inhibits the expression of apoptosis regulatory protein Bax and reduces cell apoptosis. For inflammation of the gastric mucosa, HSP70 competes for the NF-kB complex transported into the nuclear pore to inhibit the activation of NF-kB, further block the overproduction of inflammatory factors such as IL-8 and make IL-8 and NF-kB more effective. The inflammatory cascade amplification reaction is interrupted, thereby playing a role in anti-inflammatory, reducing the inflammatory damage of the gastric mucosa, protecting the gastric mucosa, and preventing the occurrence of chronic atrophic gastritis.

\section{HSP70 and CAG Treatment}

\section{Physiotherapy}

All objects (temperature higher than $-273^{\circ} \mathrm{C}$ ) can radiate infrared rays. The main thermal effect produced on the body is a series of biological effects produced through direct action and nerve reflex. In the study of Dai Jie et al. [5], the expression of HSP70 in the gastric mucosa of HSP70 and CAG rats after infrared irradiation was significantly different, and the expression rate reached $100 \%$, and the expression level was also significantly increased. It can be seen that infrared radiation can treat CAG by promoting the expression of HSP70.

\section{Chemotherapy}

Polaprezinc is often used clinically to treat CAG. Polaprezinc is a chelate of L-carnosine and zinc, which induces the production of HSPs, thereby playing an anti-ulcer effect. As a heavy metal element, zinc can activate the metal response elements present in the HSP70 promoter region [6]. Therefore, zinc is believed to induce HSP synthesis by activating HSPs gene transcription.

\section{TCM Treatment}

According to the clinical manifestations of CAG, such as poor appetite and lack of food, delayed transport and chemistry, gastric pain, gradual weight loss, etc., it belongs to the category of "fullness" and "stomach pimple" in Chinese medicine. The diseased part is in the stomach, and the spleen and stomach are the inner and outer surfaces of each other. The two rise and fall, and the other is transported and absorbed, which together complete the transport and transformation functions. External evils invade the stomach, in the initial stage, mostly in the qi component, the disease enters the blood component for a long time, resulting in poor blood flow and stasis.

The protective effect of traditional Chinese medicine on gastric mucosa is currently an active research neighborhood. Studies have found that one of the target genes of Astragalus and Panax notoginseng to treat CAG may be the HSP70 gene. In the study of Shi Rui et al. [7], Scutellaria baicalensis for replenishing qi and spleen and Panax notoginseng for promoting blood circulation and removing blood stasis acted separately. The data show that the respective effects can significantly promote the transcription of HSP70mRNA in the gastric mucosa of AG rats and promote the expression of HSP70/72 protein., Its expression level even exceeded the teprenone group. In the study of Tian Hua et al. [8], baicalein may inhibit the release of IL-8, reduce the synthesis of NF-kB and COX-2, and increase the synthesis of HSP70 to block the effect of pathogenic factors on the gastric mucosa. Chronic inflammatory damage, which has a therapeutic effect on CAG, and found that this effect is closely related to the dose of baicalein. In Zhang Huicun's studies [9], Panax notoginseng, Scutellaria baicalensis and their compatibility can significantly improve the gastric mucosal state of rats with atrophic gastritis, which may be related to the promotion of the expression of HSP70 by inducing and increasing the expression of the transcription factor GAF. C

In recent years, clinical acupuncture treatment of CAG has also achieved remarkable results. Chinese medicine believes that "gastric pain" is located in the stomach and is closely related to the foot-yangming meridian. A large number of clinical and experimental observations have confirmed that acupuncture of the foot-yangming meridian has a good repair effect on gastric mucosal injury and is a clinical treatment. The first-choice meridian for gastrointestinal disease syndrome. Zhu Xiaoxiang et al. [10] electroacupuncture Zusanli showed that this mechanical stimulus-based stimulus can promote the expression of HSP70mRNA and induce the production of HSP70 and HSP60 in gastric tissue.

Moxibustion is an important part of acupuncture and moxibustion therapy. Modern research has also shown that moxibustion has the effects of analgesia, improving blood circulation, regulating metabolic disorders, regulating immune function, and regulating viscera functions. Yi Shouxiang et al. [11] used moxibustion at the "Zusanli-Liangmen" acupoint group and non-acupoint control points to prepare a gastric ulcer model by the restrained water immersion stress method, and found that the restrained water immersion stress method can cause gastric mucosa. Injury index increased, gastric mucosa TGF- $\alpha$ content decreased, PCNA decreased, cell apoptosis index and HSP70 mRNA increased. Moxibustion at Zusanli and Liangmen can reduce gastric mucosal injury index, increase gastric mucosal TGF- $\alpha$ content, promote the expression of HSP70 mRNA and PCNA, and reduce 
gastric mucosal cell apoptosis index, which is significantly different from the model group and the control group. It indicates that moxibustion at Zusanli and Liangmen has a protective effect on the gastric mucosa of stress gastric ulcer. The mechanism may be that it promotes the synthesis of TGF- $\alpha$, stimulates the proliferation of gastric mucosal cells, and inhibits cell apoptosis. This process may be similar to moxibustion. It is related to the induction of HSP70 expression [12].

In a word, HSP70 is a kind of molecular chaperone. The study of HSP70 in different degrees of chronic atrophic gastritis, overexpression and intracellular distribution in gastric cancer will further explore its molecular mechanism for the treatment of chronic atrophic gastritis and the prevention of gastric cancer. Biological therapy provides theoretical basis. In addition, the heat shock protein peptide complex can also be used for the treatment of precancerous lesions of the digestive tract, tumor prevention and gene therapy, which is expected to improve patient survival rate, reduce trauma, greatly reduce medical expenses, and improve the quality of life.

\section{References}

1. Zhang Jing, Zhang Shiiming, Zhao Jianjun (2014) Heat shock protein 70 and brain injury [ J ]. Modern Health 18: 40.

2. You Chaoyong, Feng Yizhong (2012) Research progress on the relationship between HSP70 and malignant tumors[J]. Practical Journal of Cancer 01: 89-91+94.

3. Qiu Lie, Guan Qin (2009) The protection of HSP70 on tissue cells [J] Chongqing Medicine 38(15): 1977-1980.
4. Wu Yongmei, Ju Laiti, Xie Huizhong (2010) Related research on the expression of heat shock protein 70 (HSP70) in chronic atrophic gastritis [J]. China Journal of Modern Medicine 02: 179-182+195.

5. Dai Jie, Yang Yueping, Shao Xuehui, Bo Aihua (2005) The effect of infrared rays on the body mass and heat shock protein expression of gastric mucosal cells in atrophic gastritis rats after repair[J]. Chinese Journal of Clinical Rehabilitation 07: 70-71.

6. Yang Xiaoou, Qian Jiaming, Chen Qiang (2014) Polyprezinc induces HSP70 to protect rat gastric mucosal injury[J]. Journal of Gastroenterology and Hepatology 01: 50-53.

7. Shi Rui, Li Xiaohong, Liu Lijian, Li Jian, Li Junxiang (2012) Effects of astragalus, panax notoginseng and their compatibility on the expression of heat shock protein 70 gene in gastric mucosa of atrophic gastritis rats[J]. World Chinese Journal of Digestion 04 : 310-313.

8. Tian Hua, Wang Xiaoping, Zhang Fengli (2015) Experimental study on protective effect of baicalein on gastric mucosa in rats with chronic atrophic gastritis[J]. Bulletin of Traditional Chinese Medicine 05: 62-64.

9. Zhang Huicun, Shi Rui, Li Jian, Li Xiaohong, Li Junxiang, et al. (2013) Effects of Astragalus and Panax notoginseng on the expression of HSP70 and its transcription factor GAF in rats with atrophic gastritis[J]. World Chinese Journal of Digestion 07: 559-566.

10.Zhu Xiaoxiang, Sa Zheyan, Zheng Shuxia, Pan Xiaohua, Xu Jinsen (2015) The effect of electroacupuncture at Zusanli on HSP70mRNA in gastric tissue of rats with stress gastric ulcer[J]. Shanghai Journal of Acupuncture and Moxibustion 06: 585-587.

11. Yi Shouxiang, Yu Jie, Chang Xiaorong, et al. (2009) The effect of quercetin blocking the expression of heat shock protein 70 on moxibustion pretreatment inhibiting cell apoptosis in gastric mucosal injury [J] Chinese Journal of Information on Traditional Chinese Medicine 16(10): 30-33.

12. Yang Zhou, Chang Xiaorong, Liu MI, Peng Liang, Wen Qiong (2011) Research on anti cell injury induced by Acupuncture and moxibustion and its application[J]. Chinese Journal of emergency medicine 08: 12831285 . 